\title{
Painel de registro de medicamentos sintéticos e semissintéticos no Brasil
}

\section{Market authorization scenario for synthetic and semisynthetic drug products in Brazil}

\author{
Juçara Ribeiro Franca* \\ Ana Cerúlia Moraes Carmo (D) \\ Raphael Sanches Pereira
}

Gerência de Avaliação da Qualidade de Medicamentos Sintéticos, Agência Nacional de Vigilância Sanitária (Anvisa), Brasília, DF, Brasil

\section{* E-mail: jucara.franca@anvisa.gov.br}

Recebido: 21 jul 2020

Aprovado: 19 jan 2021

\section{RESUMO}

Introdução: 0 registro sanitário é a autorização governamental necessária para que um medicamento possa ser comercializado no Brasil. As exigências relacionadas ao registro sanitário foram reestruturadas a partir de 1999 com a criação da Agência Nacional de Vigilância Sanitária (Anvisa) e com a instituição da Política Nacional de Medicamentos Genéricos. Objetivo: Cerca de 20 anos após a implementação desta política e a criação da Anvisa, este trabalho objetivou descrever o cenário atual de registro de medicamentos sintéticos e semissintéticos, a fim de fornecer subsídios para a tomada de decisão dos agentes reguladores e regulados. Método: Para realizar o levantamento dos dados referentes aos produtos registrados, foi utilizado o Sistema de Produtos e Serviços sob a Vigilância Sanitária (Datavisa), que é de acesso exclusivo aos servidores da Anvisa. Resultados: Foram localizados 6.454 processos de registros de medicamentos sintéticos e semissintéticos válidos, que correspondem a 7.721 produtos diferentes. Mais de $80,0 \%$ dos produtos registrados são genéricos ou similares. As empresas nacionais desempenham papel importante no mercado farmacêutico brasileiro, porém ainda é possível identificar dificuldades relacionadas à inovação. Dentre as principais categorias registradas, estão os medicamentos isentos de prescrição médica, os antibacterianos de uso sistêmico, os medicamentos que atuam no sistema nervoso central, os agentes antineoplásicos e os medicamentos para tratamento de síndrome metabólica. As doenças tratadas por esses medicamentos possuem alta prevalência na população brasileira. Conclusões: Os dados apresentados demonstram a efetividade das políticas adotadas e podem ser utilizados na elaboração de novas políticas relacionadas à regularização de medicamentos no Brasil.

PALAVRAS-CHAVE: Registro; Medicamentos Genéricos; Medicamentos Similares; Medicamento Novos; Política Nacional de Medicamento Genéricos; Anvisa

\section{ABSTRACT}

Introduction: Market authorization is the government approval needed for drug product commercialization in Brazil. The requirements for market authorization were reorganized in 1999, when the Brazilian Health Regulatory Agency (Anvisa) was created and, also, when the National Police for Generic Drugs was implemented. Objective: About 20 year after the implementation of the Police and the creation of Anvisa, this work aimed to describe the current scenario of market authorization for synthetic and semisynthetic drug products. This scenario aims to aid the decision-making process of regulators and regulated. Method: We used the System of Product and Services under Health Surveilance (Datavisa) to perform the data survey regarding valid market authorizations. Access to this database is exclusive to Anvisa's employees. Results: We found 6,454 valid market authorizations, accounting for 7,721 different drug products. More than $80.0 \%$ of the market authorizations are for generic and similar drug products. The national companies play an important role in the Brazilian pharmaceutical market, but we still identified some difficulty related to innovation. Among the main approved categories, we could find several over-the-counter drug products and, also, antibacterials for systemic use, drug 
products with action in the central nervous system, antineoplasic agents, and drug product for management of metabolic syndrome. The diseases treated by these drugs are highly prevalent in Brazil. Conclusions: The data showed the effectiveness of the adopted policies and can be used in the formulation of new policies related to the regularization of drug product in Brazil.

KEYWORDS: Market Authorization; Generic Drug Products; Similar Drug Products; New Drug Application; National Police for Generic Drugs; Anvisa

\section{INTRODUÇÃO}

O mercado farmacêutico do Brasil ocupa uma posição de destaque no comércio mundial de medicamentos, podendo chegar à quinta colocação no ranking mundial até 2023. Neste ano, espera-se que este mercado movimente entre US\$39 bilhões e US\$43 bilhões, com a venda de 238 milhões de unidades ${ }^{1}$.

Para que esses produtos possam ser comercializados, é necessária a obtenção do registro sanitário. De acordo com a Lei $n^{\circ} 6.360$, de 23 de setembro de $1976^{2}$, o registro é uma "inscrição, em livro próprio após o despacho concessivo do dirigente do órgão do Ministério da Saúde, sob número de ordem, dos produtos de que trata esta Lei, com a indicação do nome, fabricante, da procedência, finalidade e dos outros elementos que os caracterizem". Em outras palavras, registro de um medicamento é a autorização de um órgão governamental especializado para que um produto seja fabricado e comercializado ${ }^{3}$. Embora a obrigação de se registrar medicamento seja anterior à Agência Nacional de Vigilância Sanitária (Anvisa), este processo foi aprimorado a partir da sua criação, por meio da Lei $n^{\circ}$ 9.782, de 26 de janeiro de $1999^{4}$.

Ainda no ano de 1999, foi implementada no Brasil a política de medicamentos genéricos ${ }^{5}$. Essa política visava estimular a concorrência comercial, melhorar a qualidade dos medicamentos e facilitar o acesso da população ao tratamento medicamentoso ${ }^{6} \mathrm{e}$ foi incorporada pela Anvisa por meio de diversos atos normativos internos. Em 2004, os atos normativos referentes ao registro de medicamentos foram redefinidos por meio da política de Regulamentação de Medicamentos no Brasil, que trouxe regras mais rígidas e maiores exigências para o registro de medicamentos. Entre outros pontos, essa política é baseada no reconhecimento de três categorias principais para o registro de medicamentos sintéticos, a saber: medicamentos novos, genéricos e similares ${ }^{7}$. Posteriormente, os medicamentos novos foram reclassificados em novos e inovadores. As definições dessas quatro categorias de produtos são encontradas atualmente na Resolução da Diretoria Colegiada (RDC) $\mathrm{n}^{\circ} 200$, de 26 de dezembro de $2017^{8}$, e estão descritas a seguir:

XXVI - medicamento genérico - medicamento similar a um produto de referência ou inovador, que se pretende ser com este intercambiável, geralmente produzido após a expiração ou renúncia da proteção patentária ou de outros direitos de exclusividade, comprovada a sua eficácia, segurança e qualidade, e designado pela DCB ou, na sua ausência, pela $\mathrm{DCl}$;

XXVII - medicamento similar - aquele que contém o mesmo ou os mesmos princípios ativos, apresenta a mesma concentração, forma farmacêutica, via de administração, posologia e indicação terapêutica, e que é equivalente ao medicamento registrado no órgão federal responsável pela vigilância sanitária, podendo diferir somente em características relativas ao tamanho e forma do produto, prazo de validade, embalagem, rotulagem, excipientes e veículos, devendo sempre ser identificado por nome comercial ou marca (Medida Provisória $n^{\circ}$ 2.190-34, de 2001);

XXVIII - medicamento novo - medicamento com Insumo Farmacêutico Ativo - IFA novo no país;

XXIX - medicamento inovador - medicamento com inovação incremental, com desenvolvimento de melhorias em relação a um medicamento já registrado no país, incluindo novos sais, isômeros ou mistura de isômeros, ésteres ou éteres de moléculas anteriormente registradas [...]

No âmbito das definições, cabe ainda incluir os medicamentos "clones", que foram criados por meio da RDC n³1, de 29 de maio de 20149. Esta Resolução instituiu o procedimento simplificado de solicitações de registro, pós-registro e renovação de registro e definiu que o medicamento "clone" é aquele cujos relatórios técnico e clínico estão vinculados aos apresentados em um processo "matriz", podendo divergir deste exclusivamente no que se refere ao nome de medicamento, layout de embalagem e informações legais presentes na bula e na rotulagem.

Ainda sobre o registro de medicamentos, é importante notar que ele é concedido com prazo definido, que pode ser de três ou de dez anos, conforme estabelecido na RDC $n^{\circ} 317$, de 22 de outubro de $2019^{10}$. As empresas detentoras dos registros de medicamentos devem solicitar frequentemente as renovações destes registros, para atualizar as suas validades. A solicitação de renovação de registro tem prazo estipulado na Lei $\mathrm{n}^{\circ}$ $6.360 / 1976^{2}$ e também na RDC $n^{\circ} 317 / 2019^{10}$. Caso um detentor de registro de medicamento não solicite a renovação tempestivamente, o registro é considerado caduco e cancelado. Além disso, outros fatos podem levar ao cancelamento do registro de um medicamento, tais como: i) infrações sanitárias da parte da detentora do registro ou da fabricante do medicamento; ii) reprovação pela Anvisa do pedido de renovação de registro por motivos técnicos ou avaliação de que o medicamento não apresenta mais benefício terapêutico que supere o risco sanitário - por exemplo, por novas descobertas relacionadas à sua eficácia, segurança ou qualidade que não eram conhecidas no momento da concessão do registro; iii) pedido da empresa detentora. Assim, pode-se dizer que os medicamentos têm um 
"ciclo de vida", que se inicia com a concessão do seu registro, passa pela realização de diversas mudanças pós-registro e termina com o seu cancelamento.

Neste contexto, cerca de 20 anos após a criação da Anvisa e da política dos genéricos e após a intensificação da regulação de medicamentos no país, o objetivo deste trabalho foi apresentar um panorama geral dos medicamentos sintéticos registrados no Brasil nas categorias de novos/inovadores, genéricos e similares, de modo a dar transparência às empresas e à sociedade sobre o trabalho da Agência e a fim de fornecer subsídios para a tomada de decisão dos agentes reguladores e regulados.

\section{MÉTODO}

Para realizar o levantamento dos dados referentes aos produtos registrados, foi utilizado o Sistema de Produtos e Serviços sob a Vigilância Sanitária (Datavisa). O Datavisa é o sistema interno de informação da Anvisa, que congrega dados sobre o cadastro de produtos e empresas, além de controlar a tramitação e o arquivo dos documentos na Agência ${ }^{11}$. Embora as informações obtidas a partir do sistema e apresentadas neste trabalho sejam de acesso público, por meio do site da Anvisa, a base de dados que consolida as informações é de acesso exclusivo aos servidores da Anvisa ${ }^{11}$.

Para este trabalho, foram considerados os registros de medicamentos válidos e, para tanto, foram examinados os produtos que protocolaram Histórico de Mudanças do Produto (HMP) nos últimos dois anos consecutivos. Essa estratégia foi adotada porque, para se obter o número de registros válidos, é necessário considerar aqueles que foram concedidos e aqueles que estão, de fato, ativos - isto é, os que não foram cancelados nem estão caducos. A declaração da caducidade ou o efetivo cancelamento podem levar algum tempo para se concretizar, de modo que uma listagem direta dos registros "não cancelados" pode apresentar um resultado superestimado por considerar registros já em fase de cancelamento ou já caducos. Por outro lado, a RDC $\mathrm{n}^{\circ} 73$, de 7 de abril de $2016^{12}$, determina que o HMP deve ser submetido anualmente, no mês de aniversário do produto, em todos os processos de registro ativos. O HMP, lista todas as mudanças realizadas ao longo do ano ou declara não ter havido mudanças pós-registro nesse mesmo período ${ }^{12}$. Assim, uma forma eficiente de estimar o número de medicamentos registrados em um ano é a listagem de todos os HMP protocolados neste ano, visto que seu protocolo é obrigatoriamente anual para os medicamentos ativos e que não haverá protocolo de HMP para medicamentos em fase de cancelamento ou com registro caduco, mesmo que o efetivo cancelamento ainda não tenha sido publicado pela Anvisa. Neste trabalho, a listagem de HMP de dois anos consecutivos foi utilizada para possibilitar a realização de dupla conferência dos dados, reduzindo erros decorrentes da falta de protocolo do documento em um ano específico e evitar a perda de produtos que eventualmente, por algum motivo, tenham tido atraso no envio do HMP em um ano.

Os medicamentos classificados como clones foram excluídos por estarem vinculados diretamente a uma matriz, não caracterizam, dessa forma, a variabilidade de medicamentos disponíveis no mercado. Em seguida, os dados foram tratados para inclusão dos produtos registrados no último ano, exclusão dos medicamentos cancelados no último ano e alteração dos titulares de registro dos produtos que solicitaram transferência de titularidade no último ano. Essa atualização foi realizada em 8 de abril de 2020.

Após levantamento inicial, os registros considerados válidos foram revisados para separação por forma farmacêutica, de modo que os processos que continham mais de uma forma farmacêutica foram divididos em dois ou mais produtos, a depender do número de formas farmacêuticas presentes. 0 motivo da divisão de produtos em formas farmacêuticas diferentes foi para equalizar os dados, visto que registros solicitados desde 11 de janeiro de 2015 são individualizados por forma farmacêutica conforme previsto na RDC $n^{\circ} 60$, de 10 de outubro de $2014^{13}$, isto é, se a divisão não fosse feita, os produtos anteriores a esta data ficariam divididos de forma diferente dos posteriores.

Os produtos com registro válido foram, então, reavaliados, para inclusão da classe terapêutica, conforme sistema de classificação Anatomical Therapeutic Chemical (ATC).

Os dados extraídos da base de dados foram organizados e tratados utilizando o software Microsoft Excel $\circledast$ versão 2002.

\section{RESULTADOS}

Foram localizados 6.454 processos de registros de medicamentos sintéticos válidos, que correspondem a 7.721 produtos diferentes. A distribuição dos produtos entre as categorias de novos/inovadores, genéricos e similares está apresentada na Tabela 1. A base de dados não permitiu que os processos de registros de medicamentos novos e inovadores fossem separados entre si. Como pode ser verificado, mais de $80,0 \%$ dos produtos registrados são cópias de outros medicamentos que já se encontravam previamente no mercado.

A distribuição de processos registrados por empresa está apresentada na Tabela 2. Pode-se verificar que cerca de $60,0 \%$ dos processos de registro ativos $(4.112 ; 63,7 \%)$ estão concentrados em 30 empresas. A maioria dessas empresas (17) são de capital nacional.

A distribuição dos produtos registrados por forma farmacêutica está apresentada na Figura 1. Como pode ser observado na Figura 1, as formas farmacêuticas mais registradas são as de uso oral, especialmente os comprimidos revestidos (1.678; $21,7 \%$ )

Tabela 1. Número de processo de registro de medicamentos sintéticos e semissintéticos por categoria regulatória.

\begin{tabular}{lcc}
\hline Categoria & Número de processos de registro & $\%$ \\
\hline Genérico & 2.819 & $43,7 \%$ \\
Similar & 2.457 & $38,1 \%$ \\
Novo & 1.178 & $18,3 \%$ \\
Total & 6.454 & $100,0 \%$ \\
\hline
\end{tabular}

Fonte: Elaborada pelos autores, 2020. 
Tabela 2. Distribuição de processo de registro de medicamentos sintéticos e semissintéticos ativos por empresa.

\begin{tabular}{|c|c|c|}
\hline Categoria & $\begin{array}{c}\text { Número do processo } \\
\text { de registro }\end{array}$ & Porcentagem \\
\hline Empresa 1 (Nacional) & 380 & $5,9 \%$ \\
\hline Empresa 2 (Nacional) & 276 & $4,3 \%$ \\
\hline Empresa 3 (Nacional) & 265 & $4,1 \%$ \\
\hline Empresa 4 (Nacional) & 234 & $3,6 \%$ \\
\hline Empresa 5 (Nacional) & 227 & $3,5 \%$ \\
\hline Empresa 6 (Nacional) & 209 & $3,2 \%$ \\
\hline Empresa 7 (Nacional) & 193 & $3,0 \%$ \\
\hline Empresa 8 (Nacional) & 185 & $2,9 \%$ \\
\hline Empresa 9 (Multinacional) & 183 & $2,8 \%$ \\
\hline Empresa 10 (Nacional) & 175 & $2,7 \%$ \\
\hline Empresa 11 (Nacional) & 172 & $2,7 \%$ \\
\hline Empresa 12 (Nacional) & 166 & $2,6 \%$ \\
\hline Empresa 13 (Nacional) & 122 & $1,9 \%$ \\
\hline Empresa 14 (Multinacional) & 122 & $1,9 \%$ \\
\hline Empresa 15 (Multinacional) & 100 & $1,5 \%$ \\
\hline Empresa 16 (Multinacional) & 96 & $1,5 \%$ \\
\hline Empresa 17 (Multinacional) & 87 & $1,3 \%$ \\
\hline Empresa 18 (Multinacional) & 84 & $1,3 \%$ \\
\hline Empresa 19 (Nacional) & 83 & $1,3 \%$ \\
\hline Empresa 20 (Nacional) & 82 & $1,3 \%$ \\
\hline Empresa 21 (Nacional) & 81 & $1,3 \%$ \\
\hline Empresa 22 (Multinacional) & 78 & $1,2 \%$ \\
\hline Empresa 23 (Nacional) & 73 & $1,1 \%$ \\
\hline Empresa 24 (Multinacional) & 70 & $1,1 \%$ \\
\hline Empresa 25 (Multinacional) & 67 & $1,0 \%$ \\
\hline Empresa 26 (Multinacional) & 64 & $1,0 \%$ \\
\hline Empresa 27 (Nacional) & 61 & $0,9 \%$ \\
\hline Empresa 28 (Multinacional) & 61 & $0,9 \%$ \\
\hline Empresa 29 (Multinacional) & 59 & $0,9 \%$ \\
\hline Empresa 30 (Multinacional) & 57 & $0,9 \%$ \\
\hline Outras empresas & 2.342 & $36,3 \%$ \\
\hline
\end{tabular}

Fonte: Elaborada pelos autores, 2020.

e comprimidos simples $(1.336 ; 17,3 \%)$. Outras formas farmacêuticas, não representadas nesta figura, somam 562 produtos $(7,3 \%)$.

As principais classificações terapêuticas identificadas para os produtos sintéticos registrados estão apresentadas na Tabela 3. Além dos medicamentos normalmente classificados como isentos de prescrição, tais como analgésicos (377; 4,9\%), majoritariamente pirazolonas (124) e anilidas (116); os anti-inflamatórios e antirreumáticos (370; 4,8\%); antifúngicos de uso dermatológico (279; 3,6\%), anti-histamínicos de uso sistêmico (251; 3,3\%), preparações para tosse e resfriado (224; $2,9 \%$ ), chama a atenção o número de registro dos antibacterianos para uso sistêmico (732; 9,5\%), em especial as fluoroquinolonas
(117) e penicilinas de amplo espectro (80); os psicoanalépticos (343; 4,4\%), com destaque inibidores seletivos da recaptação da serotonina (155); psicolépticos (299; 3,9\%), com destaque para os derivados benzodiazepínicos (89). Ainda podemos destacar os agentes antineoplásicos (298; 3,9\%) e os produtos para tratamento de síndrome metabólica (agentes que atuam no sistema renina-angiotensina (223;2,9\%), diuréticos (133; 1,7\%), agentes modificadores de lipídeos (132; 1,7\%), betabloqueadores $(124 ; 1,6 \%)$, medicamentos utilizados no diabetes (113; $1,5 \%)$ e terapia cardíaca $(93 ; 1,2 \%)$.

Os principais princípios ativos isolados e as principais associações farmacêuticas registradas estão apresentados, respectivamente, nas Figuras 2 e 3. Foram encontrados 6.374 produtos $(82,6 \%)$ contendo princípios ativos isolados e 1.347 associações medicamentosas $(17,4 \%)$. Foram identificados 886 princípios ativos isolados diferentes e 371 associações medicamentosas diferentes. Em ambos os casos, verifica-se predominância dos medicamentos de uso esporádico, em especial, dos analgésicos, antitérmicos e antimicrobianos.

\section{DISCUSSÃO}

A concessão de registros de medicamentos de uso humano é uma das atribuições da Anvisa, estabelecidas na Lei $n^{\circ}$ 9.782/19994. De acordo com os procedimentos administrativos estabelecidos pela Anvisa, os medicamentos podem ser enquadrados em diversas categorias regulatórias, incluídos medicamentos novos/ inovadores, genéricos, similares, específicos, fitoterápicos, dinamizados, gases medicinais, produtos biológicos, radiofármacos e medicamentos de baixo risco sujeitos a notificação simplificada. Os medicamentos novos/inovadores, genéricos e similares compreendem os produtos cujos insumos farmacêuticos ativos são de origem sintética ou semissintética e correspondem à maioria dos produtos regulamentados pela Anvisa ${ }^{14,15}$. Os produtos enquadrados nesta categoria são regulamentados pela RDC $n^{\circ} 200 / 2017^{8}$ e pela RDC $n^{\circ} 73 / 2016^{12}$.

Ao possibilitar a troca de medicamentos de referência por seus genéricos, a Política Nacional de Medicamentos Genéricos trouxe maior competitividade ao mercado farmacêutico, tendo se mostrado eficaz na regulação do preço dos medicamentos e favorecendo o direito de escolha do consumidor ${ }^{6}$. Não se pode negar, que, ao incentivar a concorrência de produtos com patentes expiradas, a Política Nacional de Medicamentos Genéricos foi uma grande impulsionadora da indústria nacional ${ }^{16,17}$. Como podemos observar na Tabela 2, a maioria das empresas detentoras de registro de medicamentos no Brasil são de capital nacional e muitas fazem parte de grandes grupos econômicos, que não foram listados no presente trabalho para evitar que as empresas pudessem ser identificadas. 0 crescimento das empresas nacionais, no entanto, não alterou a estrutura do mercado farmacêutico brasileiro, caracterizado como um oligopólio diferenciado $^{16}$. Os dados do presente estudo demonstram que mais da metade dos processos de registro ativos estão concentrados em 30 empresas. Tais dados corroboram com os divulgados pela Câmara de Regulação do Mercado de Medicamentos (CMED) 


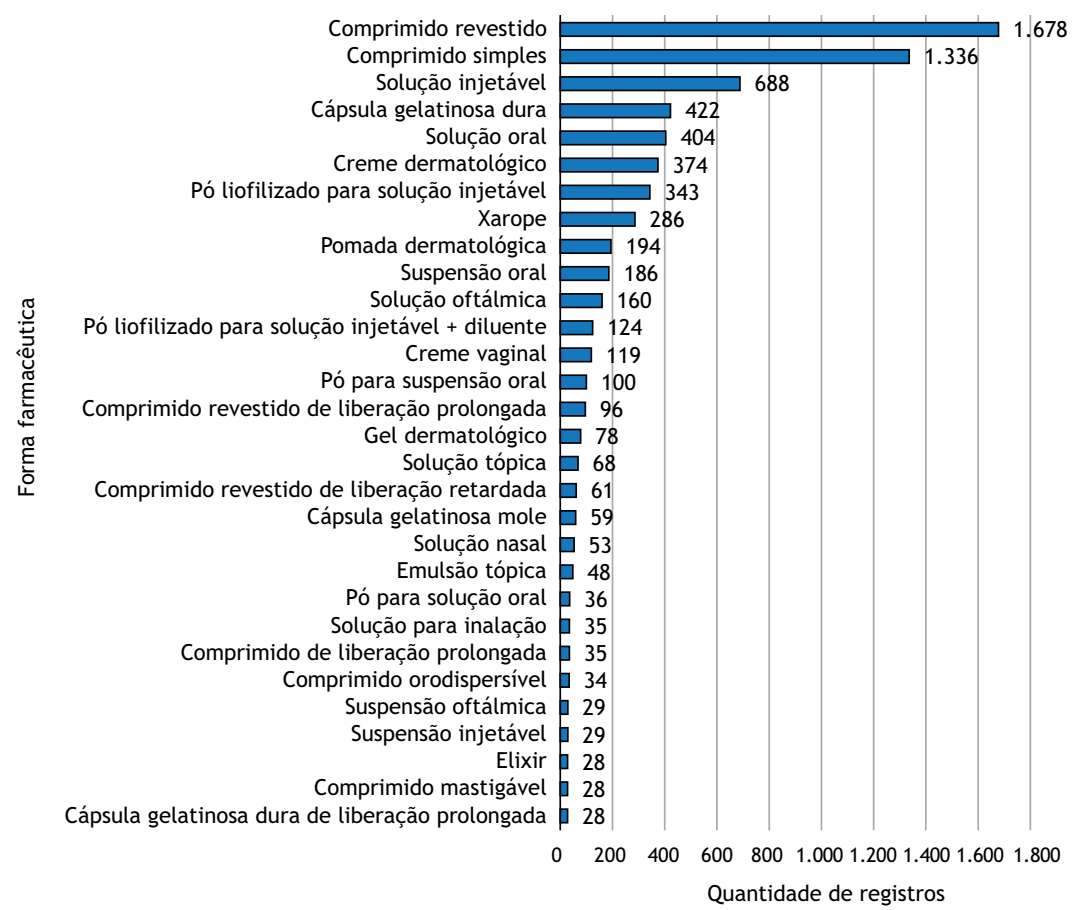

Fonte: Elaborada pelos autores, 2020.

Figura 1. Número de produtos sintéticos e semissintéticos registrados por forma farmacêutica.

Tabela 3. Principais classificações Anatomical Therapeutic Chemical (ATC) dos produtos registrados nas categorias de medicamentos sintéticos.

\begin{tabular}{|c|c|c|c|}
\hline Código ATC & Classificação ATC & $\mathrm{N}^{\circ}$ produtos registrados & $\%$ \\
\hline J01 & Antibacterianos para uso sistêmico & 732 & $9,5 \%$ \\
\hline N02 & Analgésicos & 377 & $4,9 \%$ \\
\hline M01 & Medicamentos anti-inflamatórios e antirreumáticos & 370 & $4,8 \%$ \\
\hline N06 & Psicoanalépticos & 343 & $4,4 \%$ \\
\hline N05 & Psicolépticos & 299 & $3,9 \%$ \\
\hline L01 & Agentes antineoplásicos & 298 & $3,9 \%$ \\
\hline D01 & Antifúngicos para uso dermatológico & 279 & $3,6 \%$ \\
\hline G03 & Hormônios sexuais e moduladores do sistema genital & 252 & $3,3 \%$ \\
\hline R06 & Anti-histamínicos para uso sistêmico & 251 & $3,3 \%$ \\
\hline N03 & Antiepilépticos & 230 & $3,0 \%$ \\
\hline D07 & Preparações dermatológicas contendo corticosteroides & 229 & $3,0 \%$ \\
\hline R05 & Preparações para tosse e resfriado & 224 & $2,9 \%$ \\
\hline $\mathrm{CO9}$ & Agentes que atuam no sistema renina-angiotensina & 223 & $2,9 \%$ \\
\hline S01 & Oftálmicos & 218 & $2,8 \%$ \\
\hline R03 & Agentes contra doenças obstrutivas das vias aéreas & 181 & $2,3 \%$ \\
\hline $\mathrm{A} 03$ & Medicamentos para transtornos gastrointestinais funcionais & 178 & $2,3 \%$ \\
\hline $\mathrm{A} 02$ & Medicamentos para transtornos relacionados à acidez & 164 & $2,1 \%$ \\
\hline $\mathrm{H} 02$ & Corticosteroides para uso sistêmico & 159 & $2,1 \%$ \\
\hline $\mathrm{CO3}$ & Diuréticos & 133 & $1,7 \%$ \\
\hline $\mathrm{C} 10$ & Agentes modificadores de lipídeos & 132 & $1,7 \%$ \\
\hline G01 & Anti-infecciosos e antissépticos ginecológicos & 132 & $1,7 \%$ \\
\hline J05 & Antivirais para uso sistêmico & 130 & $1,7 \%$ \\
\hline $\mathrm{CO}$ & Betabloqueadores & 124 & $1,6 \%$ \\
\hline G04 & Produtos urológicos & 117 & $1,5 \%$ \\
\hline A10 & Medicamentos utilizados no diabetes & 113 & $1,5 \%$ \\
\hline D06 & Antibióticos e quimioterápicos para uso dermatológico & 97 & $1,3 \%$ \\
\hline $\mathrm{J} 02$ & Antimicóticos para uso sistêmico & 96 & $1,2 \%$ \\
\hline $\mathrm{C} 01$ & Terapia cardíaca & 93 & $1,2 \%$ \\
\hline Outras classificações & & 1.547 & $20,0 \%$ \\
\hline Total & & 7.721 & $100,0 \%$ \\
\hline
\end{tabular}

Fonte: Elaborada pelos autores, 2020. 


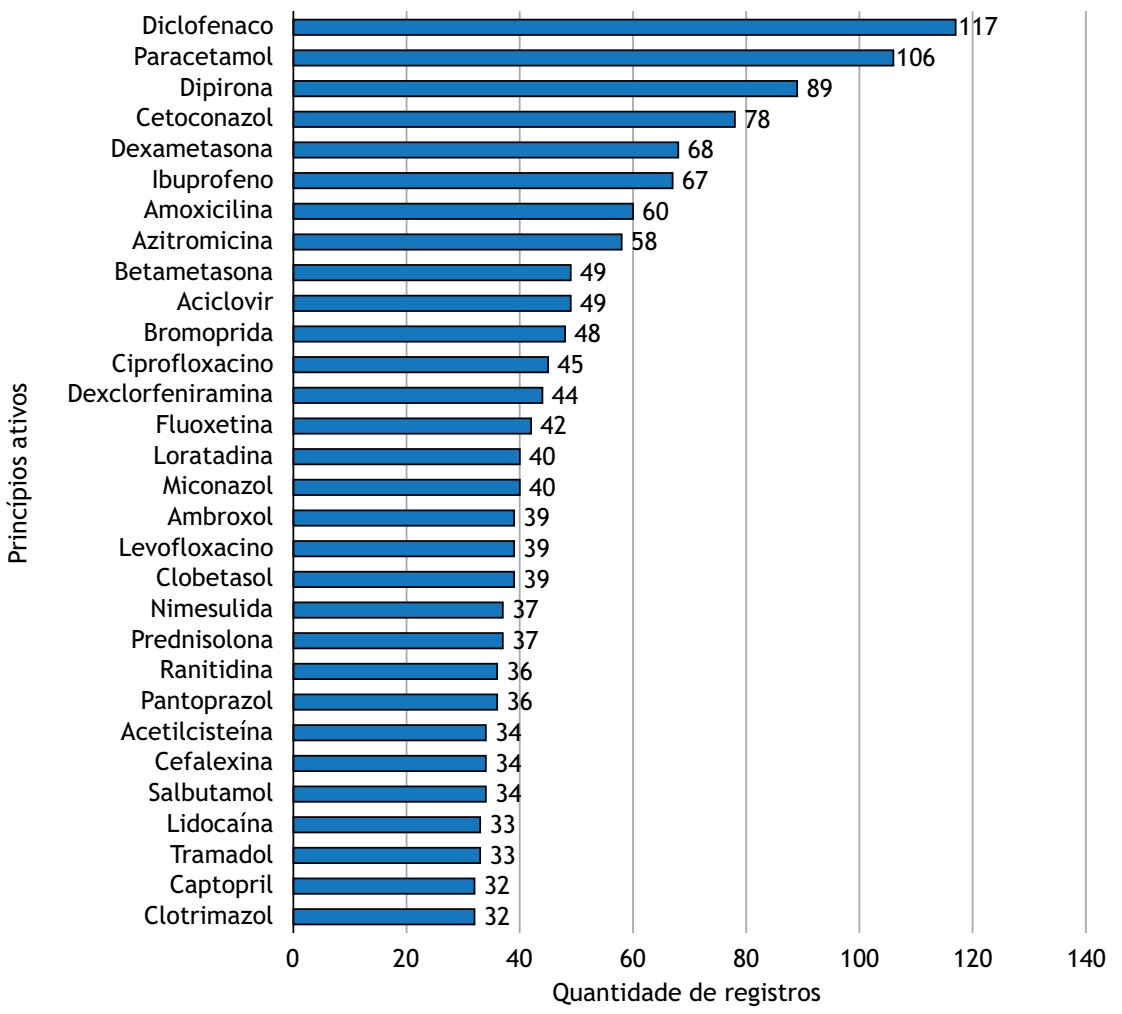

Fonte: Elaborada pelos autores, 2020.

Figura 2. Principais Insumos Farmacêuticos Ativos (IFA) isolados registrados.

Betametasona + dexclorfeniramina Cetoconazol + betametasona + neomicina Escopolamina + dipirona Cetoconazol + betametasona Sulfametoxazol + trimetoprima Amoxicilina + clavulanato Bacitracina + neomicina Etinilestradiol + gestodeno Betametasona + gentamicina Drospirenona + etinilestradiol Clioquinol + gentamicina + tolnaftato + betametasona Cafeína + orfenadrina + dipirona Nistatina + óxido de zinco Hidroclorotiazida + valsartana Etinilestradiol + levonorgestrel Tinidazol + tioconazol Hidroclorotiazida + losartana Atenolol + clortalidona Fenilefrina + clorfeniramina + paracetamol Miconazol + tinidazol

Desogestrel + etinilestradiol Adifenina + prometazina + dipirona Algestona acetofenida + enantato de estradiol Candesartana cilexetila + hidroclorotiazida Betametasona + ácido salicílico Piridoxina + dimenidrinato Piperaciclina + tazobactam Loratadina + pseudoefedrina Hidroclorotiazida + enalapril Cafeína + carisoprodol + diclofenaco + paracetamol

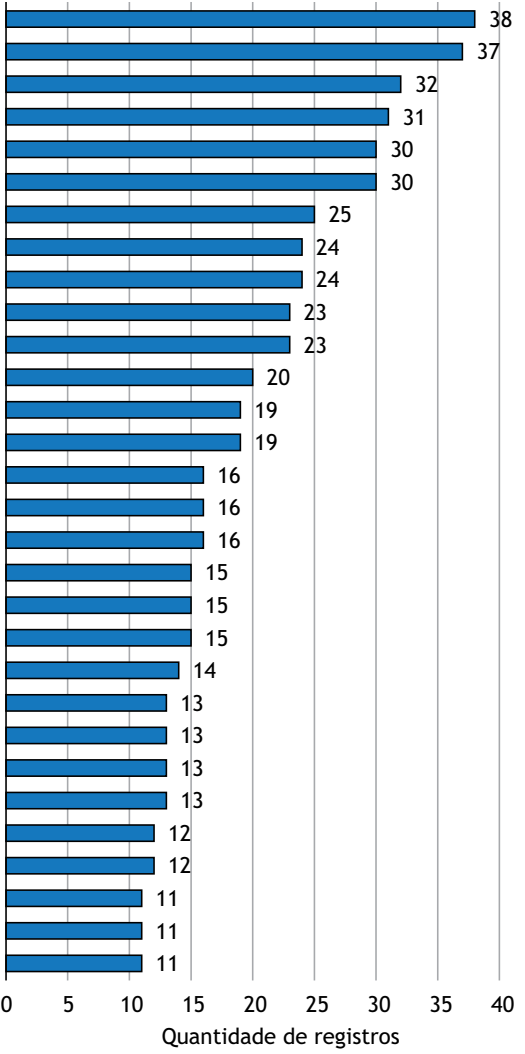

Fonte: Elaborada pelos autores, 2020.

Figura 3. Principais associações farmacêuticas registradas. 
sobre o índice de concentração de mercado, que indicam que $78 \%$ das subclasses terapêuticas comercializadas foram classificadas como fortemente concentradas ${ }^{18,19}$.

Embora tenha sido efetiva no fortalecimento da indústria farmacêutica nacional, esta política não gerou grandes estímulos à inovação no país, o que resultou nos dados observados na Tabela 1, que indicam elevado número de registros de produtos na forma de cópias. A dificuldade de inovação é também expressa nas formas farmacêuticas registradas, conforme indicam os dados apresentados na Figura 1. Formas farmacêuticas que necessitam de maior tecnologia para o seu desenvolvimento, como comprimidos e cápsulas de liberação prolongada e retardada contendo ou não microgrânulos; comprimidos orodispersíveis; adesivos transdérmicos; comprimidos mastigáveis e gomas de mascar; soluções e suspensões aerossóis; pó para inalação; implantes, dispositivo intrauterino (DIU), dispersão coloidal, correspondem a 4,1\% (313 produtos) das formas farmacêuticas registradas. Se forem retirados os comprimidos e cápsulas de liberação prolongada e retardada, encontram-se apenas 189 produtos (2,4\%). Esses dados reforçam a ausência de estímulo à inovação no mercado farmacêutico brasileiro. 0 desenvolvimento de formas farmacêuticas que diminuam o regime posológico ou que facilitem a administração é uma estratégia positiva para o aumento da adesão de pacientes ao tratamento prescrito ${ }^{20}$. Ainda assim, as formas farmacêuticas mencionadas já não seriam consideradas modernas frente ao que já é discutido em relação à medicina personalizada, como a impressão de comprimidos 3D ou cápsulas contendo dispositivos eletrônicos ${ }^{21}$.

Na Figura 1, ainda podemos observar as principais formas farmacêuticas registradas no Brasil. Em razão da facilidade de administração e das suas comodidades, os medicamentos de uso oral são os que mais se destacam. A seguir, podemos identificar os medicamentos de uso injetável, que são utilizados principalmente em ambiente hospitalar e, embora não possuam todas as comodidades dos produtos de uso oral, por vezes são a alternativa mais adequada, por apresentarem maior velocidade de ação, maior biodisponibilidade e menor susceptibilidade ao metabolismo hepático inicial (efeito de primeira passagem). Em terceiro lugar são encontrados os medicamentos de uso tópico que, em sua maioria, são utilizados para liberação local de ativos.

No que se refere às categorias terapêuticas mais registradas no país (Tabela 3), destaca-se, em primeiro lugar, antibacterianos de uso sistêmico, principalmente as fluoroquinolonas e penicilinas de amplo espectro. Dados de consumo demonstram consonância com o registro de tais classes de antimicrobianos. Em levantamento realizado pela Organização Mundial de Saúde (OMS), o Brasil apresenta uma média de uso de antibacterianos de uso sistêmico (ATC J01) correspondente a 22,75 doses por dia, superior à média europeia e de outros países americanos. As penicilinas foram o grupo de antibióticos mais frequentemente consumidos, representando $53 \%$ consumo total, enquanto as quinolonas representam $12 \%$ do consumo total desses produtos ${ }^{22}$. Os antimicrobianos de uso sistêmico também estão listados entre os principais ativos registrados, conforme pode ser verificado nas Figuras 2 e 3. Embora sejam medicamentos sob prescrição médica, até 2011 estes produtos podiam ser adquiridos em farmácias sem retenção de receita. Os dados apresentados aqui indicam a alta disponibilidade deste tipo de medicamento no mercado e reforçam a necessidade de políticas de controle do uso de antimicrobianos, tais como a $\mathrm{RDC} \mathrm{n}^{\circ} 20$, de 5 de maio de $2011^{23}$, como estratégia de prevenção à resistência antimicrobiana.

Outras categorias de destaque são os medicamentos para o tratamento do sistema nervoso central - os psicoanalépticos e os psicolépticos ocupam respectivamente a quarta e a quinta posições na Tabela 3, com destaque para a fluoxetina na Figura 2. Esses dados estão de acordo com as informações apresentadas pela OMS que indicam alta prevalência de distúrbios psiquiátricos na população brasileira. De acordo com a instituição, o Brasil lidera o ranking mundial na prevalência de transtorno de ansiedade $(9,3 \%)$ e é o quinto na prevalência de depressão $(5,8 \%)^{24,25}$. Outra classe terapêutica que merece destaque são os agentes antineoplásicos, a sexta categoria terapêutica mais registrada no país. Os dados corroboram com as informações encontradas na literatura de que o câncer é uma das principais causas de morte no Brasil ${ }^{26}$ e no mundo ${ }^{27,28}$. Ainda, pode-se destacar o registro de produtos de classes terapêuticas relacionadas ao tratamento de síndrome metabólica (antilipêmicos, anti-hipertensivos e medicamentos para tratamento do diabetes), com destaque para as associações de hidroclorotiazida a outros anti-hipertensivos (Figura 3), justificada pela alta prevalência desta síndrome na população (de 12,3\% a $44,6 \%$, na América do Sul, a depender do critério utilizado) ${ }^{29}$

Tanto na Tabela 3 como nas Figuras 2 e 3, podemos identificar a presença de diversas classes de produtos e de princípios ativos categorizados habitualmente como medicamentos isentos de prescrição (MIP). Destacamos a presença de analgésicos, como o paracetamol e a dipirona, de anti-inflamatórios, como o ibuprofeno, e de antifúngicos para o uso tópico, como cetoconazol e miconazol. Esses medicamentos são utilizados para o tratamento de sintomas comuns a diversas doenças, tais como febre, inflamação, dor de cabeça e dores em geral. De acordo com dados do IQVIA, os MIP representam 31\% do mercado farmacêutico brasileiro ${ }^{30}$. Considerando este cenário, há iniciativas sendo tomadas para simplificação de registro desses medicamentos. A principal delas é a inclusão de alguns desses produtos (medicamentos à base de paracetamol - solução, suspensão e comprimidos, nitrato de miconazol - todas as formas farmacêuticas registradas - e cetoconazol - creme) na lista de produtos de baixo risco, sujeitos a notificação simplificada, conforme sugerido na Consulta Pública $\mathrm{n}^{\circ} 819$, de $1^{\circ}$ de junho de $2020^{31}$. Como descrito previamente, esses ativos se encontram na lista de produtos com alto número de processos de registro. Essa simplificação de processo não deve ser encarada como mero estímulo a novos registros ou mesmo como uma justificativa para a redução da qualidade dos produtos. Trata-se, na verdade, de uma racionalização dos processos de trabalho, na qual a força de trabalho disponível na Anvisa será destinada para a avaliação de processos mais complexos e de maior risco sanitário. Importante esclarecer que os produtos sujeitos a notificação simplificada devem cumprir com os mesmos requisitos de qualidade estabelecidos para os medicamentos registrados. 
Entre as limitações deste estudo, podemos destacar a dificuldade em se obter dados precisos referentes aos registros válidos de medicamentos. Uma vez que uma base de dados automática não está disponível, foram realizados diversos tratamentos manuais para a obtenção dos dados. Entende-se que a disponibilização de uma base com dados mais acurados sobre os processos de registro seria benéfica para facilitar pesquisas tais como a apresentada no presente trabalho e para garantir maior transparência dos dados produzidos pela Agência para o setor produtivo e para a sociedade.

\section{CONCLUSÕES}

Os dados apresentados permitiram delinear o cenário atual de registro de medicamentos no Brasil, que foi diretamente influenciado pela Política Nacional de Medicamentos Genéricos, apresenta as empresas nacionais como protagonistas e demonstra o trabalho efetivo da Anvisa na disponibilização de medicamentos para a população brasileira. 0 cenário delineado pode ser utilizado por agentes reguladores e regulados para a proposição de novas políticas para melhoria do processo de registro de medicamentos no Brasil.

\section{REFERÊNCIAS}

1. Associação da Indústria Farmacêutica de Pesquisa Interfarma. Guia 2019 Interfarma. São Paulo: Associação da Indústria Farmacêutica de Pesquisa; 2019[acesso 16 jun 2020]. Disponível em: https://www.interfarma.org.br/ public/files/biblioteca/guia-interfarma-2019-interfarma2.pdf

2. Brasil. Lei № 6.360 , de 23 de setembro de 1976. Dispõe sobre a vigilância sanitária a que ficam sujeitos os medicamentos, as drogas, os insumos farmacêuticos e correlatos, cosméticos, saneantes e outros produtos, e dá outras providências. Diário Oficial União. 24 set 1976.

3. Said DMP. Registro sanitário de medicamentos: uma experiência de revisão [dissertação]. Rio de Janeiro: Fundação Oswaldo Cruz; 2004.

4. Brasil. Lei № 9.782, de 26 de janeiro de 1999. Define o Sistema Nacional de Vigilância Sanitária, cria a Agência Nacional de Vigilância Sanitária, e dá outras providências. Diário Oficial União. 27 jan 1999.

5. Brasil. Lei № 9.787, de 10 de fevereiro de 1999. Altera a lei № 6.360, de 23 de setembro de 1976, que dispõe sobre a vigilância sanitária, estabelece o medicamento genérico, dispõe sobre a utilização de nomes genéricos em produtos farmacêuticos e dá outras providências. Diário Oficial União. 11 fev 1999.

6. Araújo LU, Albuquerque KT, Kato KC, Silveira GS, Maciel NR, Spósito PA et al. Medicamentos genéricos no Brasil: panorama histórico e legislação. Rev Panam Salud Publica. 2010;28(6):480-92.

7. Condessa M. A política atual para a regulação de medicamentos no Brasil. Cenarium Pharma. 2005;2(1):1-34.

8. Agência Nacional de Vigilância Sanitária - Anvisa. Resolução RDC № 200, de 26 de dezembro de 2017. Dispõe sobre os critérios para a concessão e renovação do registro de medicamentos com princípios ativos sintéticos e semissintéticos, classificados como novos, genéricos e similares, e dá outras providências. Diário Oficial União. 29 jan 2018.

9. Agência Nacional de Vigilância Sanitária - Anvisa. Resolução RDC № 31, de 29 de maio de 2014. Dispõe sobre o procedimento simplificado de solicitações de registro, pós-registro e renovação de registro de medicamentos genéricos, similares, específicos, dinamizados, fitoterápicos e biológicos e dá outras providências. Diário Oficial União. 3 jun 2014.
10. Agência Nacional de Vigilância Sanitária - Anvisa. Resolução RDC №317, de 22 de outubro de 2019. Dispõe sobre os prazos de validade e a documentação necessária para a manutenção da regularização de medicamentos, e dá outras providências. Diário Oficial União. 23 out 2019.

11. Gamarski R, Mota E. Sistemas de informação em vigilância sanitária no Brasil: evolução no período de 2000 a 2005. R Eletr Com Inf Inov Saude. 2010;4(2):33-42. https://doi.org/10.3395/reciis.v4i2.678

12. Agência Nacional de Vigilância Sanitária - Anvisa. Resolução RDC № 73, de 4 de abril de 2016. Dispõe sobre mudanças pós-registro, cancelamento de registro de medicamentos com princípios ativos sintéticos e semissintéticos e dá outras providências. Diário Oficial União. 8 abr 2016.

13. Agência Nacional de Vigilância Sanitária - Anvisa. Resolução RDC № 60, de 10 de outubro de 2014. Dispõe sobre os critérios para a concessão renovação do registro de medicamentos com princípios ativos sintéticos e semissintéticos, classificados como novos, genéricos e similares, e dá outras providências. Diário Oficial União. 11 out 2014.

14. Agência Nacional de Vigilância Sanitária - Anvisa. Relatório 2016: gerência-geral de medicamentos e produtos biológicos. Brasília: Agência Nacional de Vigilância Sanitária; 2017[acesso 16 jun 2020]. Disponível em: http://portal.anvisa.gov.br/documents/33836/2946504/1\% C2\%BA+Relat\%C3\%B3rio+Gerencial+2016+-+Ger\%C3\%AAncia+ Geral+de+Medicamentos+e+Produtos+Biol\%C3\%B3gicos $/ 1$ ec 25d41-691c-46f6-a7f9-5ec8c915b7da

15. Patel $P$, Cerqueira DM, Santos GML, Soares RL, Sousa VD, Liberti L et al. A baseline analysis of regulatory review timelines for Anvisa: 2013-2016. Ther Innov Regul Sci. 2020;54(6):1428-35. https: / /doi.org/10.1007/s43441-020-00169-5

16. Gomes R, Pimentel V, Lousada M, Pieroni P, Gomes R. O novo cenário de concorrência na indústria farmacêutica brasileira. Bndes Set. 2014;(39):97-134.

17. Nishijima M, Biasoto Jr. G, Lagroteria E. A competição no mercado farmacêutico brasileiro após uma década de medicamentos genéricos: uma análise de rivalidade em um mercado regulado. Econ Soc. 2014;23(1):155-86. https://doi.org/10.1590/\$0104-06182014000100006 
18. Brasil. Resolução № 1, de 23 de fevereiro de 2015. Estabelece os critérios de composição de fatores para o ajuste de preços de medicamentos. Diário Oficial União. 2 mar 2015

19. Wertheimer A, Santella TM, Finestone A, Levy R. Clinical and economic advantages of modern dosage forms: improving medication adherence. Philadelphia: Center for Pharmaceutical Health Services Research; 2006.

20. Van Arnum P. The future of dosage forms. Pharma Tech Eur. 2014;38(1):19-22.

21. World Health Organization - WHO. WHO report on surveillance of antibiotic consumption: 2016-2018 early implementation. Geneva: World Health Organization; 2018[acesso 18 jun 2020]. Disponível em: https://apps.who.int/iris/bitstream/han dle/10665/277359/9789241514880-eng.pdf

22. Agência Nacional de Vigilância Sanitária - Anvisa. Resolução RDC № 20, de 5 de maio de 2011. Dispõe sobre o controle de medicamentos à base de substâncias classificadas como antimicrobianos, de uso sob prescrição, isoladas ou em associação. Diário Oficial União. 9 maio 2011.

23. Souza IM, Sousa JPM. Brazil: world leader in anxiety and depression rates. Rev Bras Psiquiatr. 2017;39(4):384. https://doi.org/10.1590/1516-4446-2017-2300

24. World Health Organization - WHO. Depression and other common mental disorders: global health estimates. Geneva: World Health Organization; 2017[acesso 18 jun 2020]. Disponível em: https://apps.who.int/iris/bitstream/ handle/10665/254610/WHO-MSD-MER-2017.2-eng.pdf
25. Pan American Health Organization - PAHO. Brazil. In: Pan American Health Organization - PAHO. Health in the Americas. Washington: Pan American Health Organization; 2012[acesso 17 jul 2020]. p. 129-45. Disponível em: http://www.paho.org/saludenlasamericas/index. php?option=com_docman\&task $=$ doc_view\&gid $=118 \&$ Itemid $=$

26. Nagai H, Kim YH. Cancer prevention from the perspective of global cancer burden patterns. J Thorac Dis. 2017;9(3):44851. https://doi.org/10.21037/jtd.2017.02.75

27. World Health Organization - WHO. Cancer. Fact Sheet. 2 set 2018[acesso 23 jun 2020]. Disponível em: https: / /www. who.int/news-room/fact-sheets/detail/cancer

28. Lira Neto JCG, Oliveira JFSF, Souza MA, Araújo MFM, Damasceno MMC, Freitas RWJF. Prevalence of the metabolic syndrome and its components in people with type 2 diabetes mellitus. Texto Contexto Enferm. 2018;27(3):1-8. https://doi.org/10.1590/0104-070720180003900016

29. Redação. MIPs já representam $31 \%$ do mercado farmacêutico. Panorama Farmacêutico. 18 mar 2020 [acesso 17 jul 2020]. Disponível em: https://panoramafarmaceutico.com.br/2019/03/18/mipsja-representam-31-do-mercado-farmaceutico/\#: : :text=Com mais de $1 \% 2 \mathrm{C} 25$ bilhão de unidades comercializadas e,acordo com dados da IQVIA

30. Agência Nacional de Vigilância Sanitária - Anvisa. Consulta pública $\mathrm{N}^{\circ} 819$, de 1 de junho de 2020. Proposta de revisão da resolução RDC $N^{\circ} 107$, de 2016, que aprova a lista de medicamentos de baixo risco sujeitos a notificação simplificada. Diário Oficial União. 3 jun 2020.

Agradecimentos
Os autores agradecem à Anvisa pelo fornecimento de dados.

Contribuição dos Autores

Franca JR - Concepção, planejamento do estudo (desenho do estudo), aquisição, análise, interpretação dos dados e redação do trabalho. Carmo ACM - Análise, interpretação dos dados e redação do trabalho. Pereira RS - Coleta de dados. Todos os autores aprovaram a versão final do trabalho.

Conflito de Interesse

Os autores informam não haver qualquer potencial conflito de interesse com pares e instituições, políticos ou financeiros deste estudo.

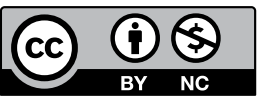

Licença CC BY-NC atribuição não comercial. Com essa licença é permitido acessar, baixar (download), copiar, imprimir, compartilhar, reutilizar e distribuir os artigos, desde que para uso não comercial e com a citação da fonte, conferindo os devidos créditos de autoria e menção à Visa em Debate. Nesses casos, nenhuma permissão é necessária por parte dos autores ou dos editores. 